\title{
Dinâmica reprodutiva e estrutura populacional de Hoplias aff. malabaricus (Bloch, 1794) (Characiformes, Erythrinidae), em açude da Bacia do Rio Taperoá, Paraíba
}

\author{
Márcio Frazão Chaves* \\ Jane Torelli \\ Carlos Henrique Targino \\ Maria Cristina Crispim \\ ${ }^{1}$ Centro de Educação e Saúde - Universidade Federal de Campina Grande \\ Olho D’Agua da Bica, s/n - CEP 58175-000 - Cuité - PB, Brasil \\ *Autor para correspondência \\ marcio_biologo@yahoo.com.br
}

Submetido em 09/04/2008

Aceito para publicação em 12/02/2009

\section{Resumo}

O presente trabalho teve como objetivo verificar a dinâmica reprodutiva e estrutura populacional da espécie Hoplias aff. malabaricus, no açude Taperoá II, localizado na Bacia do Rio Taperoá, no Cariri paraibano $\left(07^{\circ} 11^{\prime} 44^{\prime \prime} S, 07^{\circ} 13\right.$ '44'S). Foram coletados (nos meses de agosto a dezembro de 2002, janeiro a fevereiro e maio a junho de 2003) 67 indivíduos, por meio de redes de espera e tarrafa. A interferência do regime de chuvas no comportamento reprodutivo e no crescimento da espécie foi observada após a análise macroscópica das gônadas dos exemplares coletados.

Unitermos: Semi-Árido paraibano, relação peso/comprimento, grau médio de maturação gonadal

\section{Abstract}

Reproductive dynamics and population structure of Hoplias aff. malabaricus (Bloch, 1794) (Characiformes, Erythrinidae) in the dam lake of the Taperoá River Basin, Paraíba. This work aims to verify the reproductive dynamics and growth of the Hoplias aff. malabaricus species in the Taperoá II dam lake $\left(07^{\circ} 11^{\prime} 44^{\prime \prime} \mathrm{S}, 07^{\circ} 13^{\prime} 44^{\prime} \mathrm{S}\right)$, located in the basin of the River Taperoá in the Cariri region of Paraiba. Sixty-seven individuals were collected (August-December 2002, January-February, May and June 2003) by means of gillnets and cast nets. The interference of the rain regimen in the reproductive behavior and the structure of growth of the species were observed after the macrocospic analysis of the gonads of the collected units.

Key words: Paraiban semi-arido, relation length/weight, average degree of gonadal maturati 


\section{Introdução}

Nas regiões Áridas e Semi-Áridas do Nordeste brasileiro, as grandes variações dos recursos hídricos, os baixos índices pluviométricos, bem como a elevada taxa de evaporação exercem importante papel na organização e funcionalidade dos ecossistemas aquáticos. As espécies presentes nestes locais desenvolvem estratégias de sobrevivência que implicam competições intra e interespecíficas, assim como alterações na estrutura das comunidades e na disponibilidade de recursos naturais (Crispim e Watanabe, 2000). O Semi-Árido paraibano apresenta duas fases hidrológicas, uma de cheia e outra de estiagem, ambas exercendo importante papel na organização e funcionalidade dos ecossistemas regionais (Moreira, 1989).

Nesse contexto, a região do Cariri paraibano apresenta ambientes limnéticos com brusca modificação, variação do estado trófico, passando de oligotrófico a eutrófico e, mesmo, hipertrófico, em curto espaço de tempo, conforme o aumento do índice pluviométrico (Crispim e Watanabe, 2000).

A traíra, Hoplias aff. malabaricus (Bloch, 1794), em ecossistemas aquáticos como açudes e barreiros, atua como carnívoro de maior porte, exercendo o controle das populações de peixes de pequeno a médio portes (Paiva, 1974).

Como os estoques pesqueiros representam para populações humanas que vivem na região do Cariri paraibano uma importante fonte alimentar, é de grande relevância verificar os mecanismos que regulam as populações de peixes. Este trabalho tem como objetivo verificar a dinâmica reprodutiva e a estrutura populacional da espécie Hoplias aff. malabaricus correlacionando com o seu período reprodutivo em função das fortes pressões exercidas pelo regime regional de chuvas.

\section{Material e Métodos}

O açude Taperoá II (07¹ '44”S, 07¹3'44”S), integrante da Bacia do rio Taperoá, situa-se no município de Taperoá, inserido na microrregião dos Cariris Velhos, parte central do Estado da Paraíba (Moreira, 1989) (Figura 1).

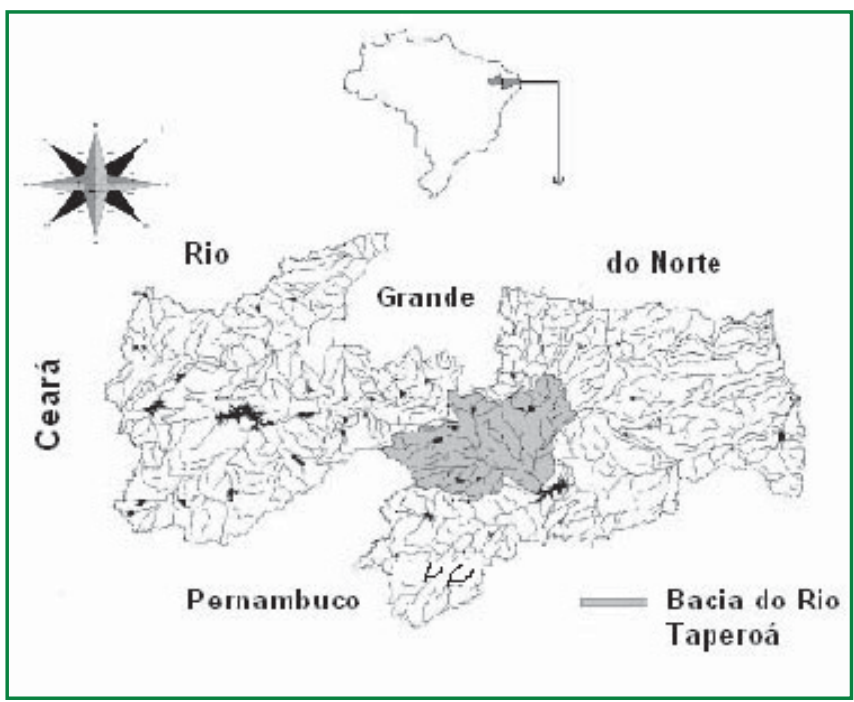

FIGURA 1: Bacia do Rio Taperoá, no Estado da Paraíba. (Fonte: Projeto Taperoá, Semi-Árido paraibano/PRODEMA/UFPB).

As coletas foram realizadas nos meses de agosto a dezembro de 2002, janeiro a fevereiro e maio a junho de 2003 durante os períodos de estiagem e chuva ocorrentes na região. Observe-se que de agosto a dezembro de 2002 a taxa média de pluviosidade foi de $20 \mathrm{~mm}$ mensais. Janeiro e fevereiro tiveram pluviosidade em torno de $100 \mathrm{~mm}$, enquanto maio e junho contaram com índice médio de chuvas de 40mm (SEMARH, 2003).

Após as coletas (em rede de espera com malhagem de $15,20,25,35$ e $40 \mathrm{~mm}$, e tarrafa com $30 \mathrm{~mm}$, entre nós adjacentes), os exemplares foram etiquetados e acondicionados em sacos plásticos, mantidos em isopor com gelo, transportados para o laboratório onde foram verificados o comprimento padrão (Ls) e peso total (Wt) para a determinação da estrutura populacional da espécie em estudo, os quais serviram de base para a determinação da relação peso/comprimento, baseada na expressão matemática $\mathbf{W t}=\mathbf{a} \mathbf{L} \mathbf{s}^{\mathbf{b}}$, com o ajustamento da curva pela equação W= $\mathbf{\Phi L} *$ (Santos, 1978; Vazzoler, 1996).

Para determinar a atividade reprodutiva da espécie foi realizada a análise macroscópica das gônadas, com posterior avaliação dos graus de maturação e identificação dos sexos (Vazzoler, 1996), sendo estes valores utilizados, em seguida, na determinação do grau médio de maturação gonadal (GM) (Santos, 1978):

$$
\mathrm{GM}=\frac{1 I+2 I I+3 I I I}{n}
$$


Onde:

GM - grau médio de maturação gonadal;

1, 2 e 3 - pesos atribuídos aos estádios I, II e III;

$\mathrm{I}$ = indivíduo imatura; II = indivíduo com gônadas em maturação e III = indivíduo com gônadas maduras;

$\mathrm{n}$ - número total de exemplares.

A época de reprodução foi determinada com base na distribuição dos valores médios do grau médio de maturação (GM) dos indivíduos coletados

\section{Resultados}

Foram amostrados 67 indivíduos de $H$. aff. malabaricus. As maiores taxas de captura ocorreram nos meses de janeiro e fevereiro/2003 (27 indivíduos), seguidas do dos meses de maio a junho/2003 (22 indivíduos) e agosto a dezembro/2002 (18 indivíduos). As médias da relação de comprimento padrão (CP) e peso total (PT) dos indivíduos coletados estão representadas nas Figuras 2, 3 e 4 . Os maiores valores dos GM foram verificados no período de maior pluviosidade da área $(100 \mathrm{~mm}$ de chuva/mês), indicando este período como a época reprodutiva da espécie. Os valores de distribuição do grau médio de maturação gonadal correlacionados com os índices pluviométricos da região estão apresentados na Figura 5.

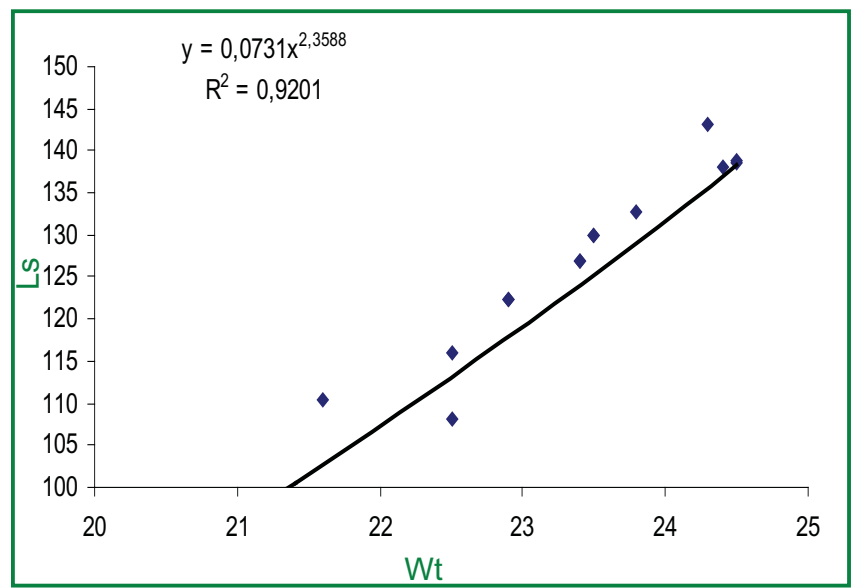

FIGURA 2: Relação entre a média do peso total (Wt) e comprimento padrão (Ls) de $H$. aff. malabaricus no Açude Taperoá II, no período de agosto a dezembro de 2002, compreendendo um regime médio $20 \mathrm{~mm}$ de chuvas ao mês.

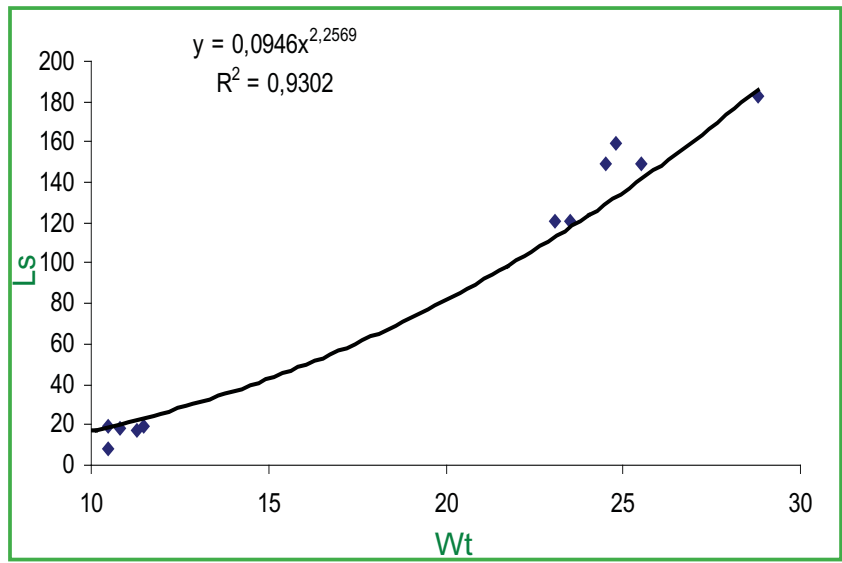

FIGURA 3: Relação entre a média do peso total (Wt) e comprimento padrão (Ls) de $H$. aff. malabaricus no Açude Taperoá II, no período de janeiro a favereiro de 2003 compreendendo um regime médio $100 \mathrm{~mm}$ de chuvas ao mês.

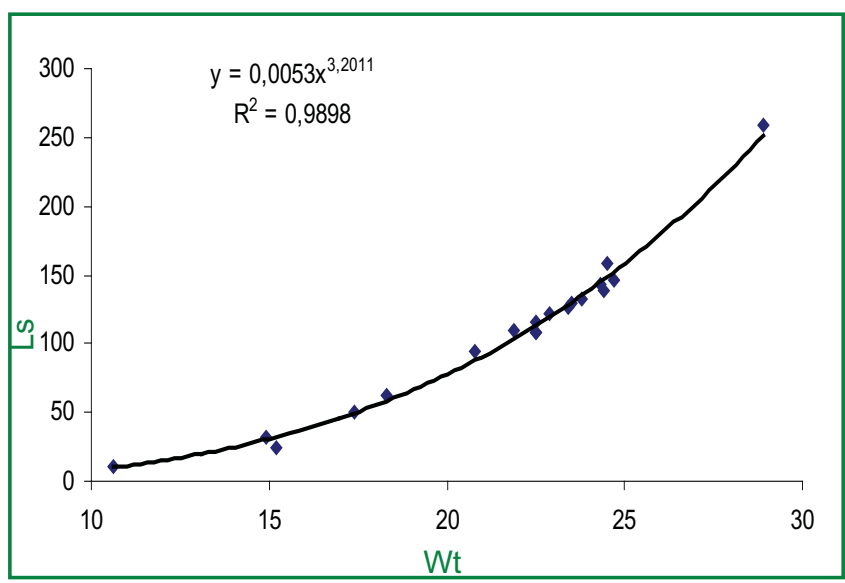

FIGURA 4: Relação entre a média do peso total (Wt) e comprimento padrão (Ls) de $H$. aff. malabaricus no Açude Taperoá II, no período de maio a junho de 2003 compreendendo um regime médio $40 \mathrm{~mm}$ de chuvas ao mês.

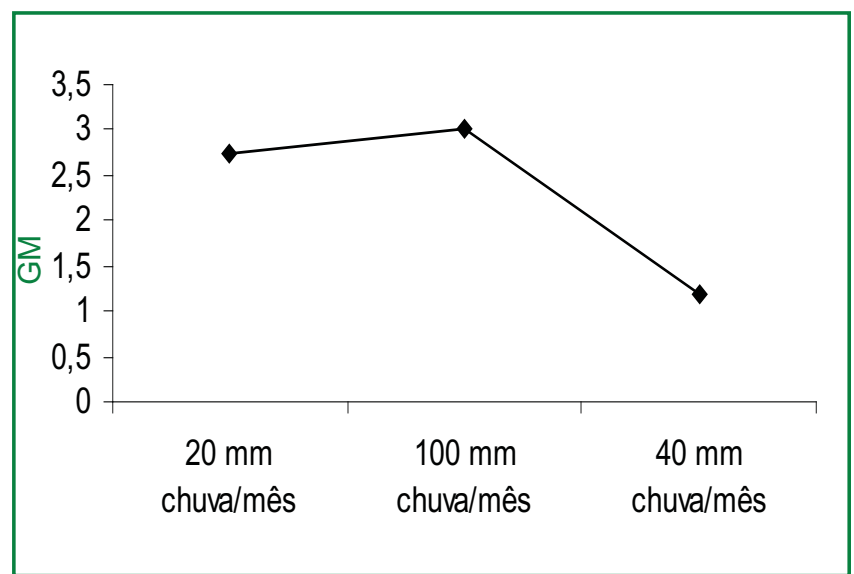

FIGURA 5: Distribuição dos valores do grau médio de maturação gonadal (GM) de H. aff. malabaricus no Açude Taperoá II durante os períodos de estiagem e chuvas da região. 


\section{Discussão}

No açude Taperoá II, as maiores médias entre relações peso total $(\mathrm{Wt}) /$ comprimento padrão (Ls) foram encontrados nos meses de maio e junho (Figura 4). Esses valores podem estar subordinados ao aumento do nível de água nos açudes em decorrência das precipitações pluviométricas registradas no período anterior (janeiro a abril - $100 \mathrm{~mm}$ de chuvas/mês). Isso corrobora o que atestam Crispim e Watanabe (2000) acerca da brusca evolução do estado trófico, como decorrência das chuvas.

A relação peso total $(\mathrm{Wt}) /$ comprimento padrão (Ls) variou de acordo com o regime regional de chuvas, apresentando os menores valores nos entre os meses de agosto a dezembro de 2002 (Figura 2) e nos meses de maio a junho de 2003 apresentando as melhores médias (Figura 4). Este decréscimo possivelmente está relacionado à baixa representatividade das gônadas e da gordura acumulada por peso total, no período estudado. O baixo peso das gônadas evidencia baixa fecundidade. Então, os menores valores da relação peso-comprimento encontrados relativo ao maior período de pluviosidade encontrado nos meses de janeiro a abril do ano de 2003 (100mm de chuvas/mês) (Figura 3) corresponde à atividade reprodutiva. Observese, porém, que a diminuição de peso pode decorrer não apenas da eliminação dos gametas, como também, a uma possível interferência do regime alimentar para as espécies, num momento em que cuidam da prole (ZavalaCamin, 1996).

Os maiores valores do grau médio de maturação gonadal (GM) concentraram-se no período onde houve maior concentração de chuvas $(100 \mathrm{~mm} / \mathrm{mês})$. Os resultados obtidos neste estudo corroboram com os encontrados por Marques et al. (2001), que estudaram o comportamento reprodutivo da espécie em um reservatório no litoral paraibano. Os referidos autores sugerem ainda, que reprodução de $H$. aff. malabaricus é possivelmente favorecida pelo aumento da oferta de alimento, da proteção e dos possíveis locais de desova nas margens da barragem, ocasionados pelo aumento no nível das águas. Trabalhos realizados por Araújo-Lima e Bittencourt, (2001) em trabalhos na Amazônia e Prado et al. (2006) em estudos no Pantanal sul, demonstram que a referida espécie inicia o seu período de desova durante as cheias. Contudo, Bialetzki et al. (2002), em estudos na planície de inundação do alto rio Paraná, verificaram um maior período de desova para a espécie no período de seca. Esta estratégia reprodutiva, segundo ele, minimiza a predação e maximiza o aproveitamento alimentar da espécie, uma vez que lhe permite alcançar estágios avançados enquanto as outras, em sua maioria estão desovando.

Segundo Vazzoler (1996), as variáveis ambientais atuam sobre os indivíduos, de modo que as condições, na época de desova, tornem-se favoráveis à sobrevivência e crescimento da prole. Assim, afirma à autora a época de desova constitui tática reprodutiva que varia de acordo com a distribuição da espécie, em função do compromisso entre a dinâmica do processo reprodutivo e da prevalência das exigências ambientais. Segundo Braga (2001), a temperatura e as precipitações pluviométricas estão entre os fatores abióticos que têm merecido maior atenção nos estudos até então realizados. Wootton (1984) demonstra que o sucesso reprodutivo de um peixe depende de onde e quando ele se reproduz e do recurso alocado para tal reprodução, devendo ocorrer no período do ano em que a produção de descendentes seja maximizada.

Paiva (1974), embora indicando que a traíra desova independentemente da época de chuvas, afirmou que o aumento da precipitação influencia no fenômeno, "definindo um período de desova de maior freqüência de indivíduos em reprodução".

\section{Agradecimentos}

Agradecemos ao DSE/CCEN/UFPB e ao Programa de Pesquisas Ecológicas de Longa Duração (PELD) e ao CNPq pela colaboração e ajuda financeira, essenciais à realização deste trabalho.

\section{Referências}

Araújo-Lima, C. A. R. M.; Bittencourt, M. M. 2001. A reprodução e o início da vida de Hoplias malabaricus (Erythrinidae: Characiformes) na Amazônia Central. Acta Amazônica, 31 (4): 693-697. 
Bialetzki, A.; Nakatani, K.; Sanches, P. V.; Baumgartner, G. 2002. Spatial and temporal distribution of larvae and juveniles of hoplias aff. Malabaricus (Characiformes, Erythrinidae) in the upper Paraná River Floodplain, Brazil. Brazilian Journal of Biology, 62 (2): 211-222.

Braga, F. M. S. 2001. Reprodução de peixes (Osteichthyes) em afluentes do reservatório de volta grande, Rio Grande, Sudeste do Brasil. Iheringia, 91: 67-74.

Crispim, M. C.; Watanabe, T. 2000. Heterogeneidade no ecossistema lacustre, baseado na comunidade zooplanctônica. Anais do V Simpósio Brasileiro de Ecossistemas, Vitória, Brasil, p.431-441.

Marques, D. K. S.; Gurgel, H. C. B.; Lucena, I. 2001. Época de reprodução de Hoplias malabaricus Bloch, 1794 (Osteichthyes, Erythrinidae) da barragem do rio Gramame, Alhandra, Paraíba, Brasil. Revista Brasileira de Zoociências, 3 (1): 61-67.

Moreira, E. R. F. 1989. Mesoregiões e microregiões da Paraíba. GAPLAN, João Pessoa, Brasil, 69pp.

Paiva, M. P. 1974. Crescimento, alimentação à salinidade e reprodução da traíra, Hoplias malabaricus (Bloch) no Nordeste brasileiro. Tese de Doutorado, Universidade Federal do Ceará, Brasil, 32pp.
Prado, C. P. A.; Gomiero, L. M.; Froehlich, O. 2006. Desova e cuidado parental em Hoplias malabaricus (Teleostei, Characiformes, Erythrinidae) no Pantanal Sul, Brasil. Brazilian Journal of Biology, 66 (2b): 697-702.

Santos, E. P. 1978. Dinâmica de populações aplicada à pesca e piscicultura. Hucitec, Ed. USP, São Paulo, Brasil, 129pp.

SEMARH. 2003. Secretaria do Meio Ambiente e dos Recursos Hídricos: Apresentação Meia Ponte. Disponível em <http:/ www.semarh.goias.gov.br/mp_coop_br_al.php>. Acesso em 20 de setembro de 2003

Vazzoler, A. E. A. M. 1996. Biologia da reprodução de peixes teleósteos: Teoria e prática. EDUEM SBI, São Paulo, Brasil, $169 \mathrm{pp}$.

Wootton, R. J. 1984. Introduction: Strategies and tacties in fish reproduction. In: Potts, G. W. \& Wooton, R. J. (Eds). Fish reproductions: Strategies and tacties. Academic Press, London, UK, p.1-12.

Zavala-Camin, L. A. 1996. Introdução aos estudos sobre alimentação natural em peixes. EDUEM, Maringá, Brasil, $129 \mathrm{pp}$. 